\title{
Magnetoelectric memory in reentrant frozen state and considerable ferroelectricity in the multiferroic spin-chain compound $\mathrm{Sm}_{2} \mathrm{BaNiO}_{5}$
}

\author{
A. Indra, ${ }^{1}$ K. Dey, ${ }^{1, *}$ S. Majumdar, ${ }^{1}$ I. Sarkar, ${ }^{2}$ S. Francoual,${ }^{2}$ R. P. Giri, ${ }^{3}$ N. Khan,${ }^{4}$ P. Mandal,${ }^{4}$ and S. Giri ${ }^{1, \dagger}$ \\ ${ }^{1}$ Department of Solid State Physics, Indian Association for the Cultivation of Science, Jadavpur, Kolkata 700032, India \\ ${ }^{2}$ Deutsches Elektronen-Synchrotron, Notkestrae 85, 22607 Hamburg, Germany \\ ${ }^{3}$ Surface Physics and Material Science Division, Saha Institute of Nuclear Physics, 1/AF - Bidhannagar, Kolkata 700064, India \\ ${ }^{4}$ CMP Division, Saha Institute of Nuclear Physics, 1/AF - Bidhannagar, Kolkata 700064, India \\ (Received 4 October 2016; revised manuscript received 14 February 2017; published 3 March 2017)
}

\begin{abstract}
We report intrinsic memory effect in magnetization and dielectricity for the spin-chain compound $\mathrm{Sm}_{2} \mathrm{BaNiO}_{5}$, pointing the cooperative glassy response below $\sim 8 \mathrm{~K}$. Signature of anomaly around $8 \mathrm{~K}$ is verified by the magnetization, heat capacity, dielectric permittivity, magnetostriction, and structural parameters as obtained from the synchrotron diffraction studies. Intriguingly, the memory effect is observed well below the magnetic and ferroelectric ordering temperatures, pointing to a reentrant frozen state. Ferroelectricity emerges above antiferromagnetic Néel temperature at $45 \mathrm{~K}$. For $4.5 \mathrm{kV} / \mathrm{cm}$ poling field the spontaneous electric polarization attains the value of $1300 \mu \mathrm{C} / \mathrm{m}^{2}$, that is the highest value in the $R_{2} \mathrm{BaNiO}_{5}$ series. Synchrotron diffraction studies confirm that ferroelectricity emerges due to structural transition from the centrosymmetric $I \mathrm{mmm}$ to a noncentrosymmetric $I m m 2$ space group. Magnetoelectric coupling is significant and scales linearly to the squared magnetization as described by the Ginzburg-Landau theory.
\end{abstract}

DOI: 10.1103/PhysRevB.95.094402

\section{INTRODUCTION}

Memory effect, the fingerprint of cooperative glassy dynamics, has been recurrently witnessed in the spin glass (SG) systems [1,2]. In the last few decades substantial theoretical and experimental efforts have been devoted for understanding the SG phenomenon [3]. It has been established that disorder and magnetic frustration attributed to the competing interactions are primarily required for the frozen SG state. In addition to the fundamental interest, the SG attracts special attention for applications to the artificial neural networks in computational science [4]. Compared to the SG, glassy dynamics involving electric dipole is rare and not well understood due to insufficient studies [5-8]. Recently, the multiglass scenario has been proposed in a few oxides and provided a new paradigm of glassiness in the dynamics of electric dipole [9-20]. In a majority of the cases glassiness in the electric dipole was proposed from the non-Arrhenius frequency response of freezing temperature. In very few cases coopoerative glassy dynamics in the electric dipoles were established from the memory effect $[11-13,16,21,22]$. In the current investigation the memory effect is observed both in dc magnetization and dielectric permittivity for a spin-chain compound $\mathrm{Sm}_{2} \mathrm{BaNiO}_{5}$.

The compound of our interest belongs to the $R_{2} \mathrm{BaNiO}_{5}$ series, which crystallizes in the orthorhombic structure with the Immm space group [23]. The $R_{2} \mathrm{BaNiO}_{5}$ series of compounds including $\mathrm{Sm}_{2} \mathrm{BaNiO}_{5}$ attract significant attention for a one-dimensional (1D) structure composed of $\mathrm{Ni}^{2+}$ $(S=1)$ chains [23]. The Ni atoms form the chain residing at the center of the flattened $\mathrm{NiO}_{6}$ octahedra along the $a$ axis. The intrachain exchange interaction is governed by

\footnotetext{
*Present address: Solid State and Structural Chemistry Unit, Indian Institute of Science, Bangalore 560012, India.

${ }^{\dagger}$ Corresponding author: sspsg2@iacs.res.in
}

the Ni-O-Ni superexchange path. On the other hand, the weaker interchain $\mathrm{Ni}-\mathrm{Ni}$ interaction is mediated through the $\mathrm{Ni}-\mathrm{O}-\mathrm{Sm}-\mathrm{O}-\mathrm{Ni}$ or Ni-O- $R$-O-Ni path. Because of $1 \mathrm{D}$ character the spin-chain compound often exhibits Haldane gap in the excitation spectrum [24-28]. The multiglass response has been reported in these spin-chain compounds [18,19]. Multiferroic order with a significant magnetoelectric coupling was also reported in a few other spin-chain compounds [28-31]. Preliminary magnetization studies were performed on $\mathrm{Sm}_{2} \mathrm{BaNiO}_{5}$ [32]. The results revealed that the zero-field-cooled (ZFC) magnetization exhibited a peak around $22 \mathrm{~K}$ along with a low temperature upturn with further decreasing temperature. Lowdimensional magnetic correlation was proposed from the high resolution Fourier transform spectroscopy on $\mathrm{Sm}_{2} \mathrm{BaNiO}_{5}$ [33]. The $\mathrm{Er}^{3+}$ spectroscopic probe proposed that the magnetic moments of $\mathrm{Sm}_{2} \mathrm{BaNiO}_{5}$ in the ordered state were polarized along the crystallographic $a$ axis [34].

In this paper we report memory effect both in dc magnetization and dielectric permittivity for $\mathrm{Sm}_{2} \mathrm{BaNiO}_{5}$, indicating cooperative glassy behavior below $\sim 8 \mathrm{~K}$. Intriguingly, the memory effect is observed much below the antiferromagnetic and ferroelectric orderings around $45\left(T_{N}\right)$ and $52 \mathrm{~K}\left(T_{C}\right)$, respectively, pointing to a reentrant frozen state. The synchrotron diffraction studies indicate that the ferroelectricity, which appears above $T_{N}$, is correlated to the structural change from the high temperature centrosymmetric $I \mathrm{mmm}$ to a noncentrosymmetric I $m m 2$ structure. The magnetization, heat capacity, dielectric permittivity, and magnetostriction studies are integrated to probe magnetic and dielectric properties. The compound exhibits considerable magnetodielectric response, which scales linearly to the squared magnetization at $10 \mathrm{~K}$ below $33 \mathrm{kOe}$, as described by the Ginzburg-Landau theory. We propose that interchain interaction at low temperature becomes significant, that is driven by the structural distortion, as evident in the striction mechanism and synchrotron diffraction studies. The Ni atoms form isosceles triangles in the $b-c$ plane at low temperature and cause topological magnetic 
frustration attributed to the non-negligible interchain interaction. This may be correlated to the intriguing reentrant frozen state.

\section{EXPERIMENTAL DETAILS}

Polycrystalline $\mathrm{Sm}_{2} \mathrm{BaNiO}_{5}$ is prepared using solid-state reaction [32]. The single phase chemical composition is confirmed by the x-ray diffraction studies at room temperature recorded in a SEIFERT $\mathrm{x}$-ray diffractometer (Model: XRAY3000P) using $\mathrm{Cu} \mathrm{K} \alpha$ radiation. The diffraction results are further confirmed by the synchrotron $\mathrm{X}$-ray diffraction studies measured at the Indian beam line of Photon Factory, Japan at a wavelength of $0.786 \AA$ in the temperature range $18-100 \mathrm{~K}$ and at $300 \mathrm{~K}$. Synchrotron $\mathrm{x}$-ray diffraction data in the temperature range $7-18 \mathrm{~K}$ are recorded at the $\mathrm{P} 09$ beamline of Petra III, Hamburg, Germany at a wavelength of $0.539 \AA$ using a 2-D Perkin Elmer detector [35]. The synchrotron powder diffraction data are analyzed using Rietveld refinement with a commercially available MAUD and FullProf softwares. The sample is pressed into a pellet, which is used for the dielectric measurements using a E4980A LCR meter (Agilent Technologies, USA) equipped with a cryogen-free cryocooler (JANIS, USA). The pyroelectric current $\left(I_{p}\right)$ is recorded in an electrometer (Keithley, model 6517B) at a constant temperature sweep rate. The $I_{p}$ is integrated over time for obtaining spontaneous electric polarization $(P)$. The poling electric fields are applied during cooling processes, and all the measurements are carried out in zero electric field during the warming mode. Before measurement of $I_{p}$ electrical connections are short circuited and waited for a sufficiently long time. For the in-field $I_{p}$ measurement magnetic field is applied during poling process and $I_{p}$ is measured in zero magnetic and electric fields. In all the measurements electrical contacts are fabricated using an air drying silver paint. Temperature dependence of heat capacity $\left(C_{p}\right)$ is measured in a physical properties measurement system (PPMS, Quantum Design). The longitudinal magnetostriction is measured by a capacitive method using a miniature tilted-plate dilatometer with an applied field parallel to the sample's length. Magnetization is measured in a commercial magnetometer of Quantum Design (MPMS, evercool) both in the ZFC and field-cooled (FC) protocols.

\section{EXPERIMENTAL RESULTS AND DISCUSSIONS}

\section{A. Magnetization and heat capacity}

Thermal variations of $\mathrm{ZFC}$ and $\mathrm{FC}$ magnetization recorded at $100 \mathrm{Oe}$ are displayed in Fig. 1(a). The results exhibit common features of the $R_{2} \mathrm{BaNiO}_{5}$ family where a broadened maximum is usually observed below the Néel temperature [32]. Here, a maximum is observed at $\sim 22 \mathrm{~K}$, which occurs due to the crystal field effect of $\mathrm{Sm}^{3+}$, as reported in the $R_{2} \mathrm{BaNiO}_{5}$ series [23]. As reported in earlier $R_{2} \mathrm{BaNiO}_{5}$ series, the signature of $T_{N}$ is difficult to detect in the thermal variation. This can be realized in the $d M / d T$ plot, as depicted in Fig. 1(b). A minimum is observed around $\sim 45 \mathrm{~K}$, indicating the Néel temperature $\left(T_{N}\right)$. Evidence of $T_{N}$ is apparent in the heat capacity $\left(C_{p}\right)$ data, as indicated by an arrow in Fig. 1(c). Another anomaly around $22 \mathrm{~K}$ is also evident in $C_{p}(T)$.
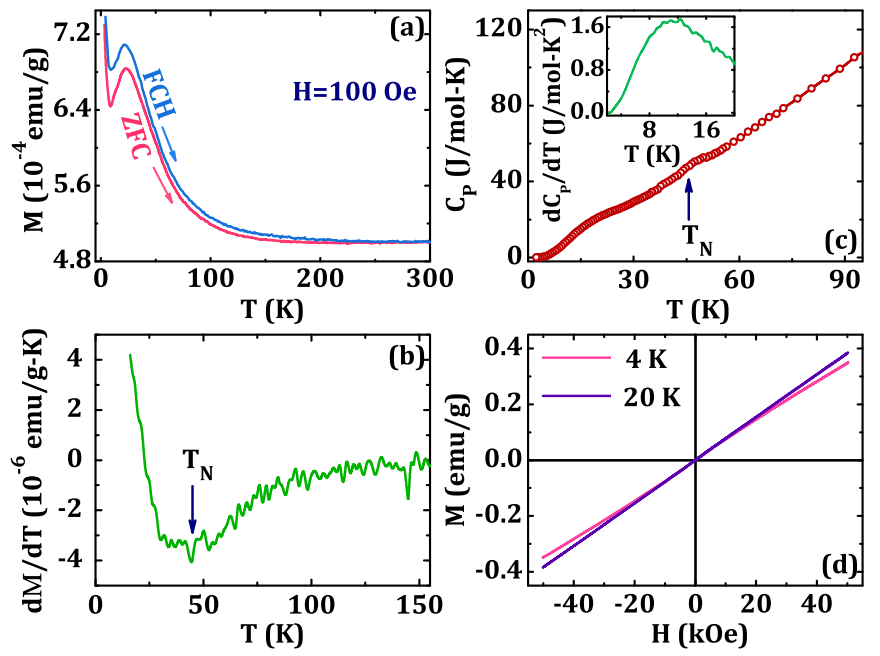

FIG. 1. Temperature ( $T$ ) variations of (a) FC-ZFC magnetization, (b) $T$ derivative of ZFC magnetization $(d M / d T)$ displaying minimum at $T_{N}$, and (c) heat capacity $\left(C_{p}\right)$. Inset of (c) shows $T$ variation of $d C_{P} / d T$ in the low $T$ region. (d) Magnetic hysteresis loops at 4 and $20 \mathrm{~K}$.

This signature is quite similar to that observed in $R_{2} \mathrm{BaNiO}_{5}$ ( $R=\mathrm{Nd}, \mathrm{Gd}$ ) [36,37], proposing rotation of $\mathrm{Ni}$ moments, which is influenced by the Sm-Ni exchange interaction and anisotropy of the $\mathrm{Sm}^{3+}$. Nearly linear magnetization curves $(M-H)$ are depicted at two representative temperatures, 4 and $20 \mathrm{~K}$ (below $T_{N}$ ), as shown in Fig. 1(d). Negligible coercivity is noted indicating soft magnetic character in accordance with that observed for the $R_{2} \mathrm{BaNiO}_{5}$ series [32]. We could not detect any convincing magnetic field induced metamagnetic transition up to $50 \mathrm{kOe}$ in the current investigation, as reported for a few members of $R_{2} \mathrm{BaNiO}_{5}$ [32].

\section{B. Spin glass}

In Fig. 1(a) we further note that both the ZFC and FC magnetization display a sharp increase below $\sim 8 \mathrm{~K}$. This signature is also observed in the $d C_{P} / d T$ data displaying a broad maximum around $8 \mathrm{~K}$, as depicted in the inset of Fig. 1(c). To understand magnetic state below $8 \mathrm{~K}$, we perform a memory experiment in the thermal variation of magnetization as described in the previous reports [38,39]. In the thermal variation temperature sweep rate is always kept fixed at $1 \mathrm{~K} / \mathrm{min}$. Herein, the $\mathrm{ZFC}$ curve recorded in the warming cycle is described as a reference curve $\left(M_{Z F C}^{\text {ref }}\right)$. In order to obtain memory curve $\left(M_{Z F C}^{\text {mem }}\right)$, sample temperature is kept fixed for $3 \mathrm{~h}\left(t_{w}\right)$ at 15 and $6 \mathrm{~K}$ during cooling in zero field. The $M_{Z F C}^{\mathrm{mem}}$ curve is recorded continuously during the warming cycle. Both the $M_{Z F C}^{\text {mem }}(T)$ and $M_{Z F C}^{\text {ref }}(T)$ curves below $30 \mathrm{~K}$ are depicted in Fig. 2(a). The difference between these two, defined as $\Delta M$, is depicted in Fig. 2(b). The $\Delta M$ vs $T$ plot shows a signature (minimum) at $6 \mathrm{~K}$, as indicated by an arrow, and reveals the memory effect as commonly noticed for the SG [1]. During the ageing process at $6 \mathrm{~K}$, the system is allowed to relax and it rearranges the spin configuration toward equilibrium. This equilibrium state is frozen with further cooling and can be retrieved during measurement on reheating. This is indicated by an anomaly in the $M_{Z F C}^{\mathrm{mem}}$ curve at $6 \mathrm{~K}$. We carefully note that 

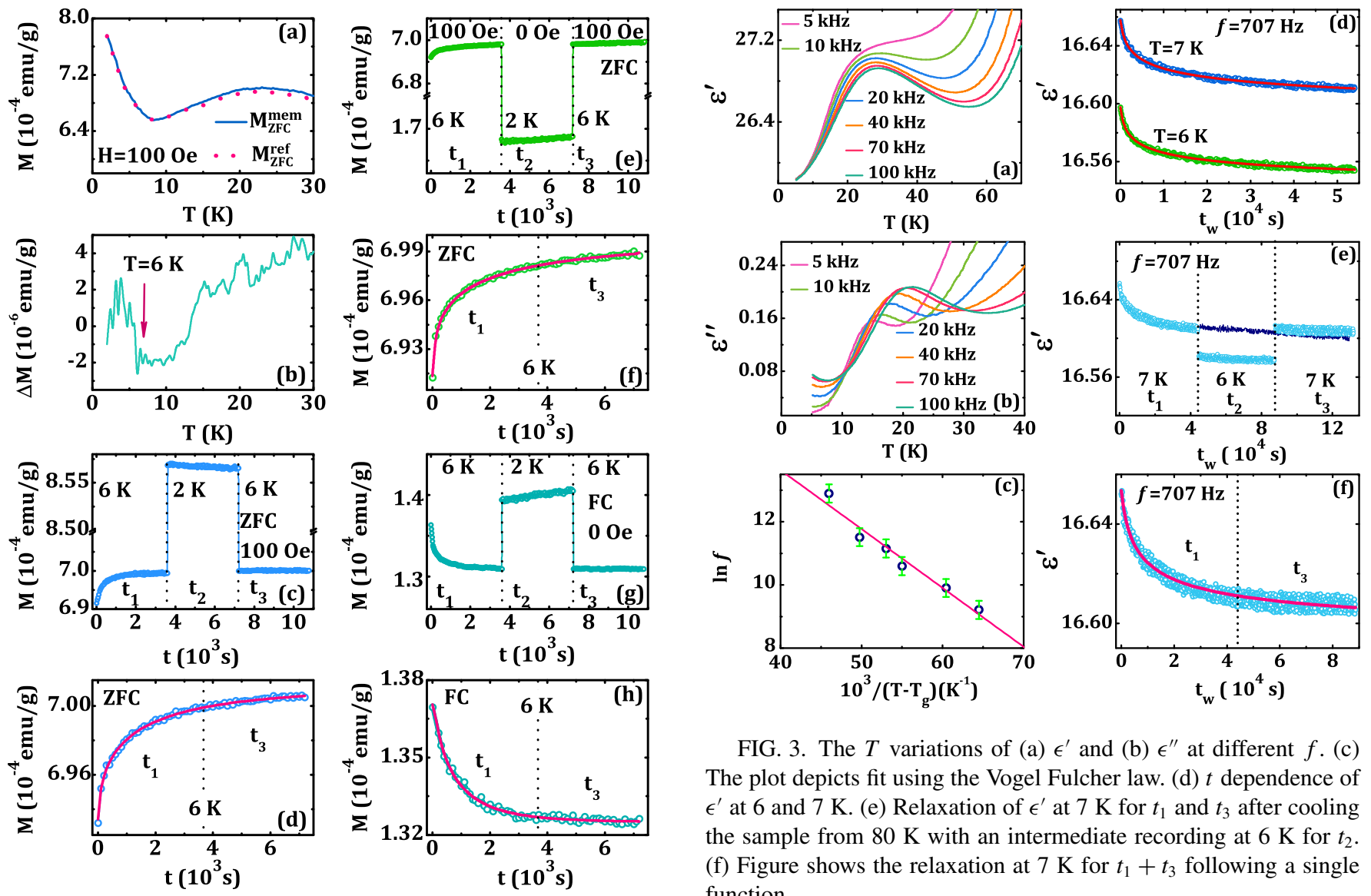

FIG. 2. (a) $T$ variations of $M_{Z F C}^{\text {ref }}$ and $M_{Z F C}^{\text {mem }}$, and (b) the difference plot $(\Delta M)$, displaying memory effect at $6 \mathrm{~K}$ as indicated by the arrow. (c) $t$ dependence of $M$ at $6 \mathrm{~K}$ for $t_{1}$, at $2 \mathrm{~K}$ for $t_{2}$, and again at $6 \mathrm{~K}$ for $t_{3}$ in 100 Oe. (e) Same $t$ dependence like (c), except for the measurement at $2 \mathrm{~K}$ for $t_{2}$, which is recorded in zero field. (d) and (f) show relaxation at $6 \mathrm{~K}$ in 100 Oe for $t_{1}+t_{3}$ following a single stretched exponential function. (g) Relaxation dynamics following protocol as in (b), except for the cooling done in FC mode. (h) shows relaxation at $6 \mathrm{~K}$ in zero field for $t_{1}+t_{3}$ following a single stretched exponential function.

any convincing signature is absent in the $M_{Z F C}^{\mathrm{mem}}$ curve at $15 \mathrm{~K}$. The memory 'dip' in the ZFC mode suggests the cooperative glassy dynamics in magnetization below the low temperature upturn.

To verify memory effect below $8 \mathrm{~K}$, further experiments on the relaxation dynamics are carried out using experimental protocols proposed by Sun et al [38]. Figures 2(c) and 2(e) show relaxation dynamics in 100 Oe at $6 \mathrm{~K}$ after cooling the sample in ZFC mode from $300 \mathrm{~K}$. At first, relaxation is recorded for $t_{1}$ at $6 \mathrm{~K}$ in $100 \mathrm{Oe}$. At the end of $t_{1}$ the sample temperature is lowered to $2 \mathrm{~K}$ and relaxation is recorded for $t_{2}$ in 100 Oe and zero field, respectively, as depicted in Figs. 2(c) and 2(e). Finally, sample temperature is heated back to $6 \mathrm{~K}$ and relaxation is recorded in 100 Oe for $t_{3}\left(t_{1}=t_{2}=t_{3}=1 \mathrm{~h}\right)$. As displayed in Figs. 2(d) and 2(f) the relaxation processes during $t_{3}$ are just the continuation of the processes after $t_{1}$. The figures depict relaxation processes recorded for $t_{1}+t_{3}$ at $6 \mathrm{~K}$ in 100 Oe, following a single stretched exponential function [40]. Similar results are observed in the relaxation dynamics

FIG. 3. The $T$ variations of (a) $\epsilon^{\prime}$ and (b) $\epsilon^{\prime \prime}$ at different $f$. (c) The plot depicts fit using the Vogel Fulcher law. (d) $t$ dependence of $\epsilon^{\prime}$ at 6 and $7 \mathrm{~K}$. (e) Relaxation of $\epsilon^{\prime}$ at $7 \mathrm{~K}$ for $t_{1}$ and $t_{3}$ after cooling the sample from $80 \mathrm{~K}$ with an intermediate recording at $6 \mathrm{~K}$ for $t_{2}$. (f) Figure shows the relaxation at $7 \mathrm{~K}$ for $t_{1}+t_{3}$ following a single function.

measured in zero field at $6 \mathrm{~K}$ after cooling the sample in FC mode ( $H=100 \mathrm{Oe}$ ) from $300 \mathrm{~K}$, as demonstrated in Fig. 2(g). Figure 2(h) displays the relaxation process in zero field for $t_{1}+t_{3}$ at $6 \mathrm{~K}$, following a single exponential function. We note that the memory effect is realized below $8 \mathrm{~K}$, which is absent above $8 \mathrm{~K}$. The overall result clearly demonstrates that relaxation dynamics after the temporary cooling $(T-\Delta T)$ or simultaneous application of field associated with $T-\Delta T$ or withdrawal of field along with $T-\Delta T$ exactly retrieves the previous history. These are the manifestations of memory effect in the relaxation dynamics. All the above experiments clearly show the spin-glass-like behavior below $8 \mathrm{~K}$, which is consistent with that observed low- $T$ behavior of $\mathrm{R}_{2} \mathrm{BaNiO}_{5}$ compounds $[18,19,30]$.

\section{Cooperative glassy behavior in dielectricity}

Dielectric permittivity $(\epsilon)$ is measured with $T$ at different frequencies $(f)$. Thermal variation of real $\left(\epsilon^{\prime}\right)$ and imaginary $\left(\epsilon^{\prime \prime}\right)$ components of $\epsilon$ are depicted in Figs. 3(a) and 3(b), respectively. In $\epsilon^{\prime}(T)$ and $\epsilon^{\prime \prime}(T)$ the signature of $T_{N}$ is absent. Any weak signature, if it exists, might be hidden in the background of broadened peak $\left(T_{f}\right)$ in the range $\sim 20-30 \mathrm{~K}\left(<T_{N}\right)$. The $T_{f}$ in both $\epsilon^{\prime}(T)$ and $\epsilon^{\prime \prime}(T)$ displays strong $f$ dispersion. The peak shift in $\epsilon^{\prime}(T)$ with $f$ can be satisfactorily analyzed by the Vogel-Fulcher (VF) law, defined by $f\left(T_{f}\right)=f_{0}\left[\exp \left(-E_{a} / K_{B}\left(T_{f}-T_{g}\right)\right)\right]$, where $f_{0}$ is the attempt frequency, $E_{a}$ is the activation energy, and $T_{g}$ is the glassy freezing temperature, the fit of which is presented 
by the solid straight line in Fig. 3(c), which provides that $T_{g}=7.6 \mathrm{~K}, f_{0}=1.36 \times 10^{9} \mathrm{hz}$, and $E_{a}=16.0 \mathrm{meV}$. The $f$ dependence of $T_{f}$ satisfying the VF law with reasonable parameters signifies the glassy freezing process [5,21], analogous to the scenario established in the spin sectors [1]. Similar to the low- $T$ increase of magnetization below $\sim 8 \mathrm{~K}$, a change of slope in $\epsilon^{\prime}(T)$ and a minimum in $\epsilon^{\prime \prime}(T)$ are noticed in Figs. 3(a) and $3(\mathrm{~b})$, respectively.

We record isothermal relaxation dynamics below $T_{g}$. During measurement the sample is cooled from $80 \mathrm{~K}$ to the desired temperatures and allowed to relax for nearly $15 \mathrm{~h}$ after stabilizing the temperatures. Despite our several attempts, we could not record $t$ dependence of $\epsilon^{\prime}$ above $8 \mathrm{~K}$. The $t$ dependence of $\epsilon^{\prime}$ at 6 and $7 \mathrm{~K}$ is depicted in Fig. 3(d) for $f=707 \mathrm{~Hz}$. The time evolution of dielectric constant is well described by a power law as

$$
\epsilon^{\prime}(\nu, t)=\epsilon^{\prime}(\nu, t=\infty)+\Delta \epsilon^{\prime}(\nu, t)\left(\frac{t_{\mathrm{w}}+t_{0}}{t_{0}}\right)^{-\alpha},
$$

where $\epsilon^{\prime}(v, t=\infty)$ is the asymptotic limit at infinite time, $\Delta \epsilon^{\prime}(v, t)$ measures the magnitude of time and frequency dependent part, and $\alpha$ characterizes the decay. The time $t_{0}$ represents the possible delay for stabilizing the sample temperature. The satisfactory fits using the above equation for 7 and $6 \mathrm{~K}$ are depicted in Fig. 3(d). The values of $\epsilon^{\prime}(\nu, t=\infty), \Delta \epsilon^{\prime}(v, t), t_{0}$ and $\alpha$ are $16.65,0.10,550 \mathrm{~s}$, and 0.13 at $7 \mathrm{~K}$, respectively, whereas those are $16.51,0.08$, $487 \mathrm{~s}$, and 0.15 , respectively, at $6 \mathrm{~K}$. The values of fitted parameters are quite consistent with that previously observed in $\beta$-hydroquinone-clathrate [41]. The aging process indicates a dielectric glassy state below $\sim 8 \mathrm{~K}$.

Similar to that observed in the relaxation dynamics of magnetization [Figs. 2(c), 2(e), and 2(g)], memory effect in $\epsilon^{\prime}$ is observed in the time dependence, as depicted in Fig. 3(e). Initially, the relaxation is recorded for $t_{1}$ at $7 \mathrm{~K}$. At the end of $t_{1}$ the sample temperature is lowered to $6 \mathrm{~K}$ and relaxation is recorded for $t_{2}$. Finally, sample temperature is reheated to $7 \mathrm{~K}$ and relaxation is recorded for $t_{3}\left(t_{1} \approx t_{2} \approx t_{3} \approx 12 \mathrm{~h}\right)$. As depicted in Fig. 3(f) the relaxation process during $t_{3}$ is just the continuation of that process after $t_{1}$ and is followed by a single function. This implies that the system can remember the state during $t_{1}$ and recalls the memory at the onset of relaxation during $t_{3}$, signifying the memory effect. Similar aging effect has been reported for nonmultiferroic poly(methyl methacrylate) thin films, pointing cooperative glassy dynamics in the dielectric response [42].

\section{Magnetodielectric}

The magnetodielectric (MD) response, defined as $\epsilon^{\prime}(H) / \epsilon^{\prime}(0)-1$, is measured with $T$, as depicted in Fig. 4(a), and $\mathrm{MD} \%$ value is consistent with some previously reported magnetodielectric materials $[17,43]$. We carefully note that the occurrence of MD does not involve magnetoresistance [44]. The MD shows a clear signature at $T_{N}$, as indicated by the arrow, pointing to magnetoelectric coupling. It also displays a maximum around $\sim 11 \mathrm{~K}$, below which the glassy behavior is observed. The plot of $M^{2}$ vs $\left[\epsilon^{\prime}(H) / \epsilon^{\prime}(0)-1\right](\%)$ at $10 \mathrm{~K}$ is depicted in Fig. 4(b). We note that the linearity holds below $\sim 33 \mathrm{kOe}$, as observed for $\mathrm{CoCr}_{2} \mathrm{~S}_{4}[45]$ as well as in $\mathrm{CoCr}_{2} \mathrm{O}_{4}$
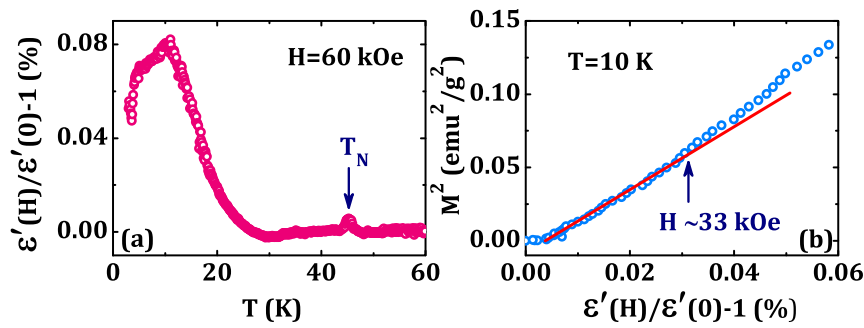

FIG. 4. (a) Percentage of MD defined as $\epsilon^{\prime}(H) / \epsilon^{\prime}(0)-1$ with $T$. (b) Plot of square of magnetization $\left(M^{2}\right)$ with $\epsilon^{\prime}(H) / \epsilon^{\prime}(0)-1$ at $10 \mathrm{~K}$

$[43,46]$. Below the ferroelectric order this plot was found linear up to $90 \mathrm{kOe}$ for $\mathrm{BiMnO}_{3}$ [47].

\section{E. Ferroelectricity}

Pyroelectric current $\left(I_{p}\right)$ is recorded with $T$ in different conditions. A peak in $I_{p}(T)$ is observed around $52 \mathrm{~K}$, as evident in Fig. 5(a). The $I_{p}(T)$ is recorded at different heating rates for $4.5 \mathrm{kV} / \mathrm{cm}$ poling field $(E)$. Nevertheless, the peaks of the three $I_{p}-T$ curves appear at almost the same temperature. The integrals of $I_{p}$ over time for three $E$ fall on a single $P(T)$ curve. This indicates that the detrapped charges, if they exist, do not contribute appreciably to the measured current. Time-integrated $I_{p}$ provides $P$ as a function of $T$, which is depicted in Fig. 5(b) for $E= \pm 4.5 \mathrm{kV} / \mathrm{cm}$. Reversal of $P$ due to a change in sign of $E$ signifies ferroelectric behavior of $\mathrm{Sm}_{2} \mathrm{BaNiO}_{5}$. Poling-field dependent polarization results are also depicted in Fig. 5 (b) from $E=1.5 \mathrm{kV} / \mathrm{cm}$ to $4.5 \mathrm{kV} / \mathrm{cm}$. We note that ferroelectric transition $\left(T_{C}\right)$ occurs at $\sim 52 \mathrm{~K}$, which is well above $T_{N}$. To confirm genuine occurrence of ferroelectricity in $\mathrm{Sm}_{2} \mathrm{BaNiO}_{5}$, we measured current using the bias electric field (BE) method as recently described by
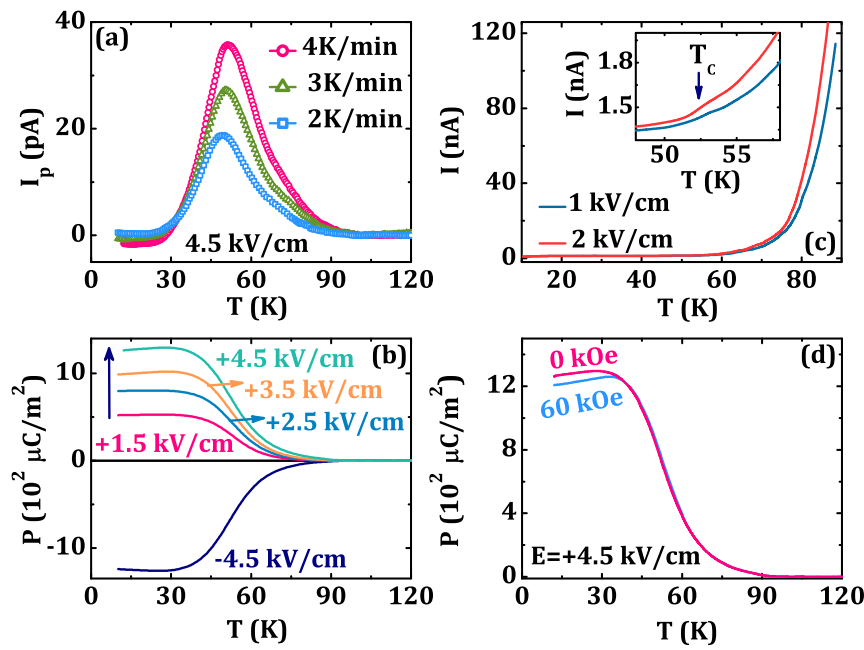

FIG. 5. (a) $T$ variations of pyroelectric current $\left(I_{p}\right)$ at different thermal sweep rates for $+4.5 \mathrm{kV} / \mathrm{cm}$ poling field. (b) $T$ variations of polarization $(P)$ for different poling fields. (c) Thermal variations of the electric current during heating under two bias electric fields following BE method. The inset of (c) highlights the FE $T_{C}$ around $52 \mathrm{~K} . T$ variations of (d) $P$ for $+4.5 \mathrm{kV} / \mathrm{cm}$ in magnetic field, $H=0$ and $60 \mathrm{kOe}$. 

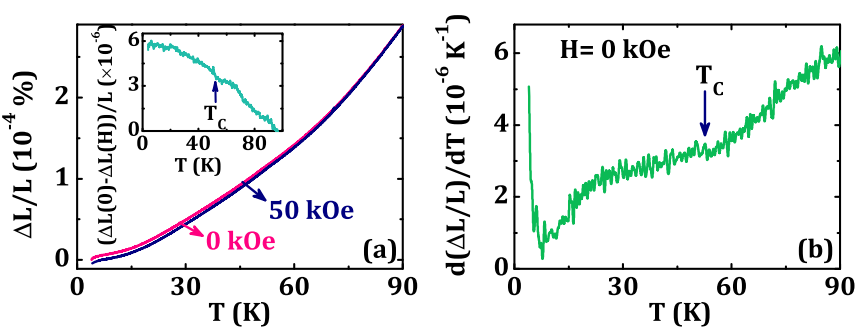

FIG. 6. Thermal variations of (a) $\Delta L / L$ in zero- field and $50 \mathrm{kOe}$, and (b) $\mathrm{d}(\Delta L / L) / \mathrm{d} T$ in zero field displaying onset of ferroelectric $T_{C}$. Inset of (a) depicts magnetostriction $[(\Delta L(0)-\Delta L(H)) / L]$ with $T$. Arrow indicates ferroelectric $T_{C}$.

N. Terada et al. [48]. The results of BE measurements are depicted in Fig. 5(c), for two bias electric fields. The inset of Fig. 5(c) clearly shows anomaly in the current data around $52 \mathrm{~K}$, at which the peak is observed in the pyroelectric current. The signature at $\sim 52 \mathrm{~K}$ in both the pyroelectric and $\mathrm{BE}$ measurement indicates genuine occurrence of ferroelectricity $[48,49]$. The ferroelectricity in $\mathrm{Sm}_{2} \mathrm{BaNiO}_{5}$ is consistent with that observed for the Haldane spin chain compound $\mathrm{Er}_{2} \mathrm{BaNiO}_{5}$ [31], proposing that the short range magnetic order induced ferroelectricity above $T_{N}$. Importantly, the value of $P$ in the current investigation is $\sim 1300 \mu C / \mathrm{m}^{2}$ for $E=$ $4.5 \mathrm{kV} / \mathrm{cm}$, which is largest among the reported spin chain compounds $[28,29,31,50]$. The significant change in electric polarization is observed below $T_{N}$ due to magnetic field, as shown in Fig. 5(d). This further indicates that magnetoelectric coupling appears below $T_{N}$.

\section{F. Magnetostriction}

The temperature dependences of thermal expansion $(\Delta L / L)$ in zero field and in field are recorded. The results are displayed in Fig. 6(a). It shows a slope change close to ferroelectric $T_{C}$ which is evident in the derivative plot as shown in Fig. 6(b). A sharp minimum is also evident in the figure, below which glassy behavior is observed. This result indicates that origin of ferroelectricity is correlated to the striction mechanism, involving contravention of space inversion symmetry [51]. To confirm it, the synchrotron diffraction studies are carried out, which is described below. In Fig. 6(a) the $\Delta L / L(T)$ is also displayed in $50 \mathrm{kOe}$. The difference between $\Delta L / L(T)$ in zero and in field, as shown in the inset of Fig. 6(a), is described as magnetostriction $(\Delta L(0)-\Delta L(H)) / L$ and reveals an increasing trend with decreasing temperature. The weak signature of $T_{C}$ in $(\Delta L(0)-\Delta L(H)) / L$ is indicated by the arrow. Magnetic field driven short range ordering might have a significant role at $T_{C}$, as suggested in $\mathrm{Er}_{2} \mathrm{BaNiO}_{5}$ [31], which is manifested in $(\Delta L(0)-\Delta L(H)) / L(T)$.

\section{G. Structural studies}

$\mathrm{Sm}_{2} \mathrm{BaNiO}_{5}$ crystallizes in an orthorhombic structure with a centrosymmetric space group $I \mathrm{mmm}$ at room temperature. Neutron diffraction studies have been performed in the isomorphous series of compounds [32]. The majority of the $R_{2} \mathrm{BaNiO}_{5}$ family orders with a magnetic structure characterized by the $(1 / 2,0,1 / 2)$ propagation vector. If the same magnetic structure is considered for $\mathrm{Sm}_{2} \mathrm{BaNiO}_{5}$, magnetic structure does not break the space inversion symmetry to account for the ferroelectricity.

To find out the origin of ferroelectricity, the structural properties are investigated by the x-ray powder diffraction studies using a synchrotron source over a low temperature range, $7-100 \mathrm{~K}$ and at $300 \mathrm{~K}$. Example of a diffraction pattern together with the refinement with $1 \mathrm{mmm}$ space group at $300 \mathrm{~K}$ is shown in Fig. 7(a) with coordinates of $\mathrm{Sm}(0.5,0,0.2049)$, $\mathrm{Ba}(0.5,0.5,0), \mathrm{Ni}(0,0,0), \mathrm{O} 1(0.5,0,0), \mathrm{O} 2(0,0.130$, 0.151 ), and lattice constants, $a=3.8032(6), b=5.8649(4)$,
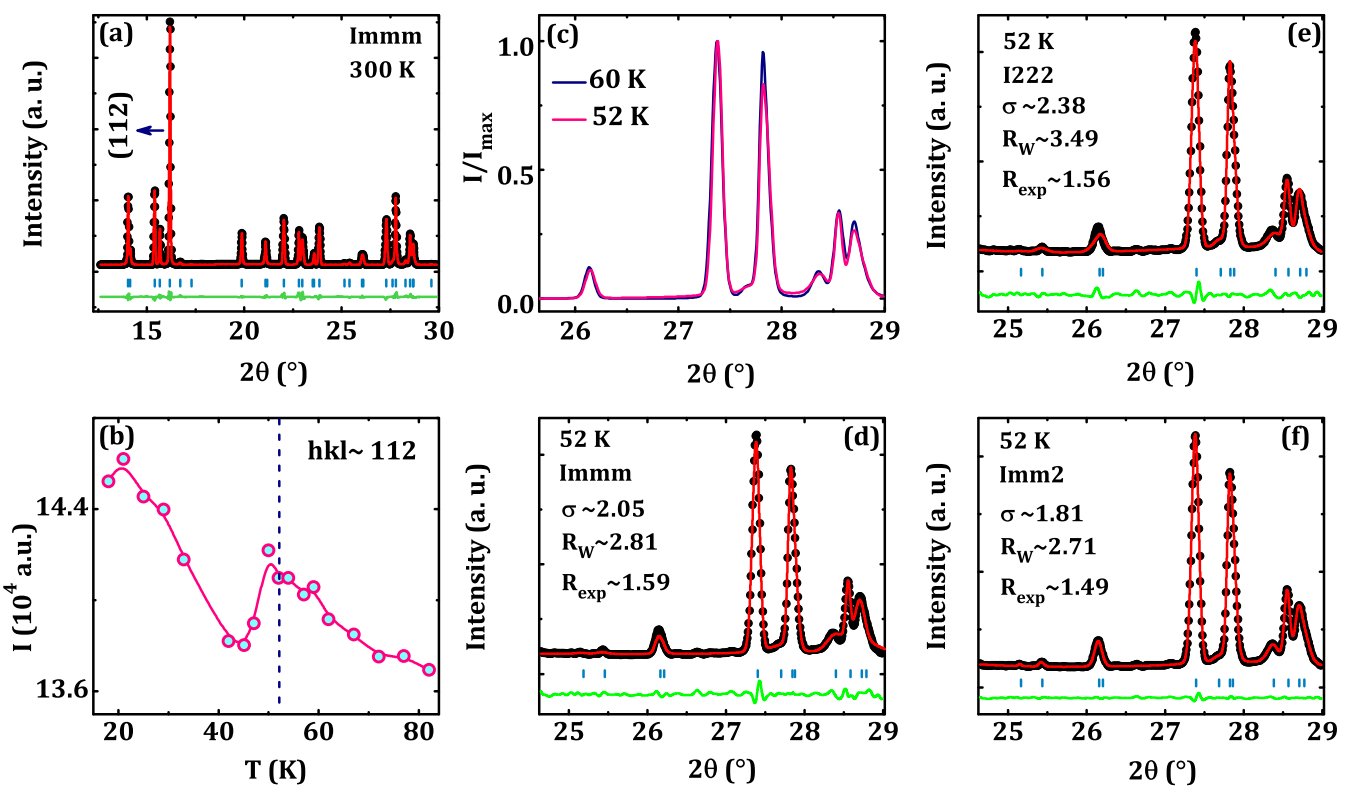

FIG. 7. (a) The Rietveld refinement of x-ray powder diffraction pattern (symbols) at $300 \mathrm{~K}$. The solid curve demonstrates the fit. (b) $T$ variation of the integrated intensity of $\left(\begin{array}{lll}1 & 1 & 2\end{array}\right)$ peak. (c) Normalized x-ray diffraction pattern at 60 and $52 \mathrm{~K}$. Refinement of diffraction pattern at $52 \mathrm{~K}$ with (d) $I m m m$, (e) $I 222$, and (f) $I m m 2$ space groups. The reliability parameters are shown in the inset of the figures. 

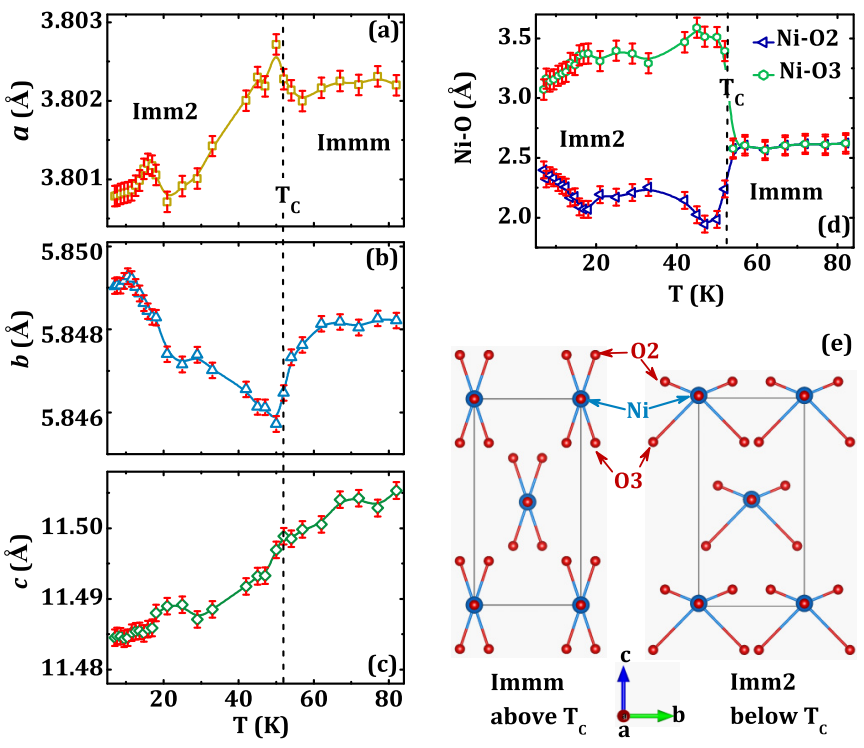

FIG. 8. Variations of [(a)-(c)] lattice parameters and (d) $\mathrm{Ni}$ O2/Ni-O3 $\left(d_{\mathrm{Ni}-\mathrm{O} 2} / d_{\mathrm{Ni}-\mathrm{O} 3}\right)$ bond lengths with $T$. (e) Figure shows schematic representation of structure displaying distortion of $\mathrm{NiO}_{6}$ octahedra below and above the structural transition.

and $c=11.5325(3) \AA$. The reasonable reliability parameters are $R_{w}(\sim 2.30), R_{\exp }(\sim 1.09)$, and $\sigma(\sim 2.10)$ at $300 \mathrm{~K}$. The bars below the diffraction pattern represent the diffraction peak positions and the difference plots are shown at the bottom for all the refinements, as shown in Figs. 7(a), 7(d), 7(e), and $7(\mathrm{f})$. Temperature variation of the integrated intensity of the (112) diffraction peak is depicted in Fig. 7(b), which displays a step-like change near FE $T_{C}$. This intensity change is $\sim 2.8 \%$, which is much larger than the results observed for other ferroelectric materials $[45,52,53]$. A selective $2 \theta$ region of the diffraction patterns at FE $T_{C}(52 \mathrm{~K})$ and above $T_{C}$ at $60 \mathrm{~K}$ are compared in Fig. 7(c). We further note that the diffraction pattern at $52 \mathrm{~K}$ could not be refined satisfactorily using centrosymmetric Immm space group, as depicted in Fig. 7(d). We propose that a structural transformation from $1 \mathrm{mmm}$ to a noncentrosymmetric structure involves the occurrence of ferroelectricity. To address this issue, we incorporate the AMPLIMODE [54] and ISODISTORT [55] softwares to locate possible space group below FE $T_{C}$. Out of all possible noncentrosymmetric structures, $I 222$ and $I \mathrm{~mm} 2$ space groups are feasible. The fits of the diffraction pattern with $I 222$ and $I m m 2$ space groups are depicted in Figs. 7(e) and 7(f), respectively. We note that the best fit is realized for Imm 2 space group with coordinates of $\operatorname{Sm} 1(0.5,0,0.2589), \mathrm{Sm} 2$ $(0.5,0,0.8470), \mathrm{Ba}(0.5,0.5,0), \mathrm{Ni}(0,0,0.0064), \mathrm{O} 1$ $(0.5,0,0.0188), \mathrm{O} 2(0,0.3308,0.1045), \mathrm{O} 3(0,0.5696$, 0.7796), and lattice constants, $a=3.8022(7), b=5.8464(7)$, $c=11.4988(6) \AA$. The reliability parameters are given in the insets of Figs. 7(d), 7(e), and 7(f) for comparison. In addition, the best fit using I $\mathrm{mm} 2$ space group is evident by the difference plot at the bottom of the diffraction pattern.

The $T$ variations of lattice parameters, as obtained from the fitted data, are depicted in Figs. 8(a)-8(c). A sharp change around FE $T_{C}$ is observed in $a(T)$ and $b(T)$, which is not so apparent in $c(T)$. The thermal variations of Ni-O2 $\left(d_{\mathrm{Ni}-\mathrm{O} 2}\right)$ and Ni-O3 $\left(d_{\mathrm{Ni}-\mathrm{O} 3}\right)$ bond lengths are depicted in Fig. 8(d). A step-like decrease and increase are observed in $d_{\mathrm{Ni}-\mathrm{O} 2}$ and $d_{\mathrm{Ni}-\mathrm{O} 3}$, respectively, around $\mathrm{FE} T_{C}$. It indicates that distortion of $\mathrm{NiO}_{6}$ octahedra is significant around $\mathrm{FE} T_{C}$. This observation is consistent with the previously reported theoretical prediction [31]. In $\mathrm{NiO}_{6}$ octahedra, $\mathrm{O} 1$ occupies the apex position along the $\mathrm{Ni}$-chain, whereas positions of basal oxygen, defined as $\mathrm{O} 2 / \mathrm{O} 3$, distort for the $\operatorname{Imm} 2$ space group. The distortions of $\mathrm{NiO}_{6}$ octahedra for structural change from $I \mathrm{mmm}$ to $I \mathrm{~mm} 2$ space group are depicted in Fig. 8(e). Apart from this, an anomaly in the integrated intensity [Fig. 7(b)] and lattice parameters [Figs. 8(a)-8(c)] are observed around $22 \mathrm{~K}$, at which an apparent signature is observed in $M(T)$ and $C_{p}(T)$. This has been proposed due to the crystal field effect of $\mathrm{Sm}^{3+}$, as reported in $R_{2} \mathrm{BaNiO}_{5}$ series [23]. It, further, indicates a significant magnetoelastic coupling near $22 \mathrm{~K}$.

\section{DISCUSSION AND CONCLUSION}

The reentrant multiglass behavior in magnetic and dielectric response, as confirmed from the magnetoelectric memory effect, is an intriguing issue for $\mathrm{Sm}_{2} \mathrm{BaNiO}_{5}$. For $\mathrm{Dy}_{2} \mathrm{BaNiO}_{5}$, the reentrant magnetic glassy response has been addressed from the peculiar magnetic structure $[18,19]$. In the current investigation we propose a fresh perception behind this multiglass response. In order to investigate possible structural correlation to the observed magnetoelectric glassy behavior below $8 \mathrm{~K}$, the synchrotron diffraction studies are extended down to $7 \mathrm{~K}$. Thermal variations of $\mathrm{Ni}-\mathrm{Ni}\left(d_{\mathrm{Ni}-\mathrm{Ni}}\right)$ distance along $c$ and $b$ axes are obtained from the refined co-ordinates as depicted in Figs. 9(a) and 9(b), respectively. Although sufficient data are not available below $8 \mathrm{~K}$ due to the constrain in the experimental facility, the decreasing trend is apparent as indicated in $d_{\mathrm{Ni}-\mathrm{Ni}}(T)$ as well as in $a(T), b(T)$, and $c(T)$. This change is further supported by the $\Delta L / L(T)$ plot. The anomalous decreasing trend in $\Delta L / L(T)$ below $\sim 8 \mathrm{~K}$ is depicted in Fig. 9(c). These results indicate that the glassy
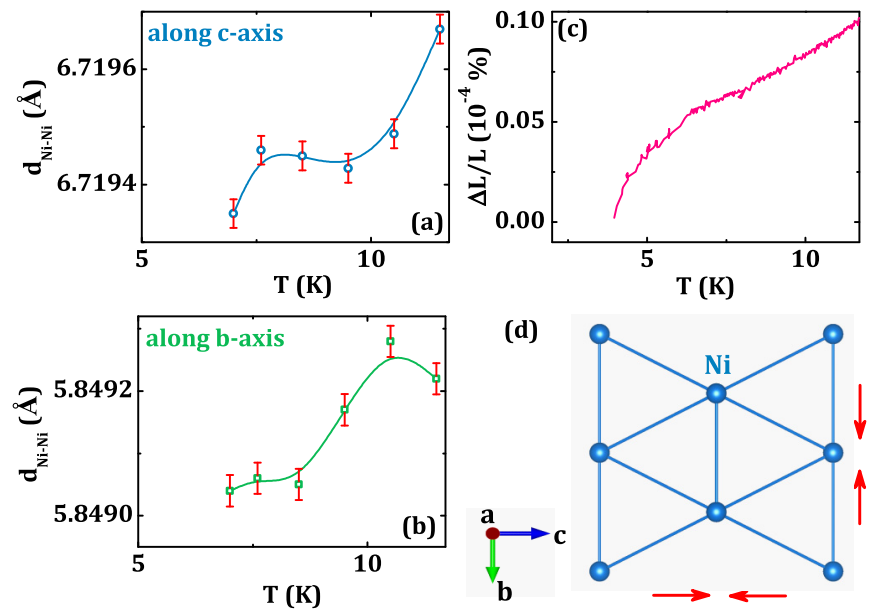

FIG. 9. $T$ variations of $\mathrm{Ni}-\mathrm{Ni}\left(d_{\mathrm{Ni}-\mathrm{Ni}}\right)$ bond length along (a) $c$ axis and (b) $b$ axis, (c) $\Delta L / L(T)$. (d) Schematic representation of nearest neighboring $\mathrm{Ni}$ atoms forming distorted $2 D$ triangular lattices in the $b-c$ plane. The arrows indicate the contraction of $d_{\mathrm{Ni}-\mathrm{Ni}}$ along $b$ and $c$ axes. 
behavior is correlated to these structural changes. Due to this thermal contraction below $8 \mathrm{~K}$, the interchain $\mathrm{Ni}-\mathrm{Ni}$ distance or $d_{\mathrm{Ni}-\mathrm{Ni}}$ in the $b$-c plane is also contracted as indicated by the arrows in Fig. 9(d). This thermal contraction may bring out significant interchain interactions. Here, the $\mathrm{Ni}^{2+}$ ions form the edge sharing triangular lattices in the $b$ - $c$ plane, as shown schematically in Fig. 9(d). The edge sharing triangles formed by the $\mathrm{Ni}^{2+}$ ions at $7 \mathrm{~K}$ are the isosceles triangles, as confirmed from the refined coordinates. Since neutron results are not available for $\mathrm{Sm}_{2} \mathrm{BaNiO}_{5}$, the nature of exchange interaction is not clearly understood. Nevertheless, the antiferromagnetically coupled edge sharing isosceles triangles carry necessary ingredient for the topological magnetic frustration [56-58]. In the current investigation the edge sharing isosceles triangles formed by the antiferromagnetically coupled $\mathrm{Ni}^{2+}$ ions may give rise to the glassy behavior driven by the topological magnetic frustration.

Another salient feature of this study is the observed intricate multiferroic order in $\mathrm{Sm}_{2} \mathrm{BaNiO}_{5}$. In fact, the multiferroic properties have been investigated for a few members of the $R_{2} \mathrm{BaNiO}_{5}$ series [28-31]. Herein, we confirm that ferroelectric transition appears well above $T_{N}$ and is correlated to the structural transition. This is confirmed from the synchrotron diffraction studies. The magnetization studies in the thermal variations clearly depict that the ZFC and FC magnetization do not coincide at $T_{N}$. Intriguingly, those coincide around $\sim 250 \mathrm{~K}$, which is much above $T_{N}$ at $45 \mathrm{~K}$. The results clearly demonstrate that short range magnetic order significantly dominates above $T_{N}$. Although the occurrence of ferroelectricity is addressed from the structural transition to a noncentrosymmetric $\operatorname{Imm} 2$ space group from the centrosymmetric $I \mathrm{mmm}$ space group, the correlation of ferroelectricity to the dominant short range magnetic ordering cannot be ruled out. In fact, the appearance of ferroelectricity was proposed to be driven by the short range magnetic order for
$\mathrm{Er}_{2} \mathrm{BaNiO}_{5}$ [31]. The magnetoelectric coupling is confirmed when electric polarization is recorded in magnetic field. Importantly, the magnetoelectric coupling is missing just below ferroelectric $T_{C}$ and is detected only below the long range magnetic ordering temperature. The results further conclude that ferroelectric polarization can be tuned by the magnetic field below $T_{N}$, where the magnetoelectric coupling coexists with the symmetry of the magnetic structure. The direct measurements of the spin structure by scattering methods like neutron or the resonant $\mathrm{x}$-ray scattering could be conclusive for the complete understanding of the multiferroic order in $\mathrm{Sm}_{2} \mathrm{BaNiO}_{5}$. Detailed studies will be performed in the future.

This study highlights two salient features of the reentrant frozen state and the observed intricate multiferroic order. The signature of cooperative glassy response is manifested through the memory effect both in magnetization and dielectric permittivity below $\sim 8 \mathrm{~K}$. Topological frustration driven by antiferromagnetically coupled triangular lattices is suggested for the occurrence of reentrant glassy response. Ferroelectricity appears above the antiferromagnetic Néel temperature. The synchrotron diffraction studies confirm that ferroelectricity appears due to structural transformation from the centrosymmetric Immm to a noncentrosymmetric Imm2 space group. The observed spontaneous electric polarization in $\mathrm{Sm}_{2} \mathrm{BaNiO}_{5}$ is found to be the largest in the $R_{2} \mathrm{BaNiO}_{5}$ family. The compound reveals considerable magnetoelectric response below the antiferromagnetic ordering temperatures.

\section{ACKNOWLEDGMENTS}

S.G. acknowledges the SERB Project No. SB/S2/CMP029/2014 and DST, India for the financial support to perform experiment at KEK, Tsukuba, Japan for synchrotron diffraction studies. A.I. wishes to thank INSPIRE, DST India for the fellowship.
[1] J. A. Mydosh, Spin Glasses: An Experimental Introduction (Taylor \& Francis, London, 1993).

[2] S. F. Edwards and P. W. Anderson, J. Phys. F 5, 965 (1975).

[3] K. Binder and A. P. Young, Rev. Mod. Phys. 58, 801 (1986).

[4] D. J. Amit, H. Gutfreund, and H. Sompolinsky, Phys. Rev. A 32, 1007 (1985).

[5] A. Karmakar, S. Majumdar, S. Banerjee, and S. Giri, Europhys. Lett. 92, 57009 (2010).

[6] Z. Nussinov, I. Vekhter, and A. V. Balatsky, Phys. Rev. B 79, 165122 (2009).

[7] I. Raičević, J. Jaroszyński, D. Popović, C. Panagopoulos, and T. Sasagawa, Phys. Rev. Lett. 101, 177004 (2008).

[8] S. Pankov and V. Dobrosavljević, Phys. Rev. Lett. 94, 046402 (2005).

[9] V. V. Shvartsman, S. Bedanta, P. Borisov, W. Kleemann, A. Tkach, and P. M. Vilarinho, Phys. Rev. Lett. 101, 165704 (2008).

[10] W. Kleemann, S. Bedanta, P. Borisov, V. V. Shvartsman, S. Miga, J. Dec, A. Tkach, and P. M. Vilarinho, Eur. Phys. J. B 71, 407 (2009).
[11] K. Singh, A. Maignan, Ch. Simon, V. Hardy, E. Pachoud, and C. Martin, J. Phys.: Condens. Matter 23, 126005 (2011).

[12] K. Singh, A. Maignan, Ch. Simon, S. Kumar, C. Martin, O. Lebedev, S. Turner, and G. Van Tendeloo, J. Phys.: Condens. Matter 24, 226002 (2012).

[13] S. Kumar, K. Singh, M. Miclau, Ch. Simon, C. Martin, and A. Maignan, J. Solid State Chem. 203, 37 (2013).

[14] D. Choudhury, P. Mandal, R. Mathieu, A. Hazarika, S. Rajan, A. Sundaresan, U. V. Waghmare, R. Knut, O. Karis, P. Nordblad, and D. D. Sarma, Phys. Rev. Lett. 108, 127201 (2012).

[15] D. Choudhury, S. Mukherjee, P. Mandal, A. Sundaresan, U. V. Waghmare, S. Bhattacharjee, R. Mathieu, P. Lazor, O. Eriksson, B. Sanyal, P. Nordblad, A. Sharma, S. V. Bhat, O. Karis, and D. D. Sarma, Phys. Rev. B 84, 125124 (2011).

[16] Y. Yamaguchi and T. Kimura, Nat. Commun. 4, 2063 (2013).

[17] S. Sharma, T. Basu, A. Shahee, K. Singh, N. P. Lalla, and E. V. Sampathkumaran, Phys. Rev. B 90, 144426 (2014).

[18] T. Basu, P. L. Paulose, K. K. Iyer, K. Singh, N. Mohapatra, S. Chowki, B. Gonde, and E. V. Sampathkumaran, J. Phys.: Condens. Matter 26, 172202 (2014). 
[19] T. Basu, N. Mohapatra, K. Singh, and E. V. Sampathkumaran, AIP Adv. 5, 037128 (2015).

[20] S. S. Rathore and S. Vitta, Sci. Rep. 5, 9751 (2015).

[21] A. Karmakar, K. Dey, S. Chatterjee, S. Majumdar, and S. Giri, Appl. Phys. Lett. 104, 052906 (2014).

[22] K. Taniguchi, N. Abe, S. Ohtani, and T. Arima, Phys. Rev. Lett. 102, 147201 (2009).

[23] E. García-Matres, J. L. Martínez, and J. Rodríguez-Carvajal, Eur. Phys. J. B 24, 59 (2001).

[24] A. Zheludev, J. M. Tranquada, T. Vogt, and D. J. Buttrey, Phys. Rev. B 54, 7210 (1996).

[25] A. Zheludev, E. Ressouche, S. Maslov, T. Yokoo, S. Raymond, and J. Akimitsu, Phys. Rev. Lett. 80, 3630 (1998).

[26] A. Zheludev, S. Maslov, T. Yokoo, S. Raymond, S. E. Nagler, and J. Akimitsu, J. Phys.: Condens. Matter 13, R525 (2001).

[27] S. Raymond, T. Yokoo, A. Zheludev, S. E. Nagler, A. Wildes, and J. Akimitsu, Phys. Rev. Lett. 82, 2382 (1999).

[28] K. Singh, T. Basu, S. Chowki, N. Mahapotra, K. K. Iyer, P. L. Paulose, and E. V. Sampathkumaran, Phys. Rev. B 88, 094438 (2013).

[29] S. Chowki, T. Basu, K. Singh, N. Mohapatra, and E. V. Sampathkumaran, J. Appl. Phys. 115, 214107 (2014).

[30] T. Basu, K. Singh, N. Mohapatra, and E. V. Sampathkumaran, J. Appl. Phys. 116, 114106 (2014).

[31] T. Basu, V. V. Ravi Kishore, S. Gohil, K. Singh, N. Mohapatra, S. Bhattacharjee, B. Gonde, N. P. Lalla, P. Mahadevan, S. Ghosh, and E. V. Sampathkumaran, Sci. Rep. 4, 5636 (2014).

[32] E. García-Matres, J. L. García-Muñoz, J. L. Martínez, and J. Rodríguez-Carvajal, J. Magn. Magn. Mater. 149, 363 (1995).

[33] Yu. A. Hadjiisldi, I. V. Paukov, and M. N. Popova, Phys. Lett. A 189, 109 (1994).

[34] S. A. Klimin, A. S. Galkin, and M. N. Popova, Phys. Lett. A 376, 1861 (2012).

[35] J. Strempfer, S. Francoual, D. Reuther, D. K. Shukla, A. Skaugen, H. Schulte-Schrepping, T. Kracht, and H. Franz, J. Synchrotron Radiat. 20, 541 (2013).

[36] E. A. Popova, S. A. Klimin, M. N. Popova, R. Klingeler, N. Tristan, B. Büchner, and A. N. Vasilév, J. Exp. Theor. Phys. 118, 611 (2014).

[37] E. A. Popova, R. Klingeler, N. Tristan, B. Büchner, and A. N. Vasiliev, Phys. Rev. B 85, 174402 (2012).
[38] Y. Sun, M. B. Salamon, K. Garnier, and R. S. Averback, Phys. Rev. Lett. 91, 167206 (2003).

[39] H. Khurshid, P. Lampen-Kelley, Ó. Iglesias, J. Alonso, M. Phan, C.-J. Sun, M.-L. Saboungi, and H. Srikanth, Sci. Rep. 5, 15054 (2015).

[40] D. De, A. Karmakar, M. K. Bhunia, A. Bhaumik, S. Majumdar, and S. Giri, J. Appl. Phys. 111, 033919 (2012).

[41] M. C. Rheinstädter, K. Knorr, and H. Rieger, Phys. Rev. B 69, 144427 (2004).

[42] K. Fukao and A. Sakamoto, Phys. Rev. E 71, 041803 (2005).

[43] N. Mufti, A. A. Nugroho, G. R. Blake, and T. T. M. Palstra, J. Phys.: Condens. Matter 22, 075902 (2010).

[44] G. Catalan, Appl. Phys. Lett. 88, 102902 (2006).

[45] K. Dey, A. Karmakar, A. Indra, S. Majumdar, U. Rütt, O. Gutowski, M. V. Zimmermann, and S. Giri, Phys. Rev. B 92, 024401 (2015).

[46] T. D. Sparks, M. C. Kemei, P. T. Barton, R. Seshadri, E.-D. Mun, and V. S. Zapf, Phys. Rev. B 89, 024405 (2014).

[47] T. Kimura, S. Kawamoto, I. Yamada, M. Azuma, M. Takano, and Y. Tokura, Phys. Rev. B 67, 180401(R) (2003).

[48] N. Terada, Y. S. Glazkova, and A. A. Belik, Phys. Rev. B 93, 155127 (2016).

[49] C. De, S. Ghara, and A. Sundaresan, Solid State Commun. 205, 61 (2015).

[50] G. Nénert and T. T. M. Palstra, Phys. Rev. B 76, 024415 (2007).

[51] J. Bertinshaw, C. Ulrich, A. Günther, F. Schrettle, M. Wohlauer, S. Krohns, M. Reehuis, A. J. Studer, M. Avdeev, D. V. Quach, J. R. Groza, V. Tsurkan, A. Loidl, and J. Deisenhofer, Sci. Rep. 4, 6079 (2014).

[52] K. Dey, S. Majumdar, and S. Giri, Phys. Rev. B 90, 184424 (2014).

[53] K. Dey, A. Karmakar, S. Majumdar, and S. Giri, Phys. Rev. B 87, 094403 (2013)

[54] D. Orobengoa, C. Capillas, I. Aroyo, and J. M. Perez-Mato, J. Appl. Crystallogr. 42, 820 (2009).

[55] B. J. Campbell, H. T. Stokes, D. E. Tanner, and D. M. Hatch, J. Appl. Crystallogr. 39, 607 (2006).

[56] J. E. Greedan, J. Mater. Chem. 11, 37 (2001).

[57] S. T. Bramwell, S. G. Carling, C. J. Harding, K. D. M. Harris, B. M. Kariuki, L. Nixon, and I. P. Parkin, J. Phys.: Condens. Matter 8, L123 (1996).

[58] A. P. Ramirez, Annu. Rev. Mater. Sci. 24, 453 (1994). 